\title{
Kristobalitbildung $\left(\mathrm{SiO}_{2}\right)$ im Herstellungsprozess überlastfester Differenzdrucksensoren
}

Dipl.-Ing. S. Hildebrandt, Prof. Dr. L. Alff, Institut für Material- und Geowissenschaften, Technische Universität Darmstadt, Petersenstr. 23, 64287 Darmstadt, shildebrandt@oxide.tu-darmstadt.de

Dipl.-Ing. T. Kober, Prof. Dr.-Ing. R. Werthschützky Institut für Elektromechanische Konstruktionen, Technische Universität Darmstadt, Merckstr. 25, 64283 Darmstadt, t.kober@emk.tu-darmstadt.de

\section{Kurzbeschreibung}

Im Herstellungsprozess überlastfester Differenzdrucksensoren werden Glaswafer durch Wärmebehandlung definiert umgeformt. Dieser Temperaturprozess begünstigt das Kristallwachstum auf der Oberfläche des Glaswafers. Das Kristallwachstum fördert die Bildung von Mikrorissen im Glas. Es werden keine zusätzlichen passivierenden Beschichtungen benötigt, die während der Temperaturbehandlung mit dem Glaswafer chemisch reagieren oder mechanische Spannungen durch Fehlanpassung des Temperaturausdehnungskoeffizienten gegenüber dem Glaswafer erzeugen könnten.

Das beschriebene Verfahren ermöglicht dagegen das Umformen von Glaswafern ohne Beeinträchtigung der Qualität der Waferoberfläche. Das Kristallwachstum kann durch eine evakuierte Prozessumgebung während des Temperaturschritts soweit reduziert werden, dass für den beschriebenen Prozesszeitraum von $5 \mathrm{~h}$ bei $700{ }^{\circ} \mathrm{C}$ durch Röntgendiffraktogramme sowie AFM-Analyse kein Kristobalitwachstum nachgewiesen werden kann. Dies ermöglicht die direkte Weiterbearbeitung der Wafer z.B. für das Waferfügen durch Anodisches Bonden.

\section{Einleitung und Motivation}

In der Prozessmesstechnik werden zur messtechnischen Auswertung von Füllständen und Volumenströmen verbreitet Differenzdrucksensoren genutzt. Diese müssen gegenüber statischer als auch dynamischer Drucküberlastung robust sein. Um den geforderten Volumenstrom innerhalb eines pneumatischen oder hydraulischen Leitungssystems aufrecht zu erhalten, ist es häufig notwendig, einen statischen Systemdruck im Leitungssystem aufzubauen, der den zu messenden Differenzdruck um mehrere Größenordnungen übersteigt. Die Messelemente selbst weisen lediglich eine auf ca. 1 bar begrenzte Überlastfestigkeit auf. Es werden daher Überlastschutzmechanismen benötigt, die die Empfindlichkeit und die Messunsicherheit des Messelementes nicht beeinträchtigen. Dies wird nach dem Stand der Technik durch Edelstahlmembranen mit definierter Ölfüllung realisiert. Für den Nennmessbereich von $\Delta p=10$ mbar ermöglichen Differenzdrucksensoren, die feinwerktechnische Schutzmechanismen nutzen, eine Überlastfestigkeit bis $\Delta p=160$ bar.

Ein mikromechanisch integrierter Überlastschutz reduziert die Komplexität der feinwerktechnischen Konstruktionen im Vergleich zum Stand der Technik (Abb. 1). Mit dem überlastfesten Differenzdrucksensor mit Nennmessbereich $\Delta p=10$ mbar ist es gegenwärtig möglich eine mittlere Überlastfestigkeit von $\Delta p=$ 220 bar zu erreichen. Das vorliegende neuartige Messelement erreicht diese Eigenschaften für einseitige Überlastung, indem die Auslenkung der Messplatte begrenzt wird. Dieser im Messelement selbst integrierte Überlastschutz aus strukturiertem SCHOTT BOROFLOAT ${ }^{\circledR} 33$ schützt die Siliziummessplatte vor statischer Drucküberlastung (Abb. 1).

\subsection{Homogene Gegenlager aus Glas}

Die mittlere Tiefe der bereits hergestellten asphärischen Gegenlager im Glasgegenkörper beträgt $12 \mu \mathrm{m}$ [1]. Für die Herstellung von Gegenlagern mit einer Strukturtiefe von $12 \mu \mathrm{m}$ sind die entscheidenden Parameter die Waferhöhe, die Umformtemperatur sowie die Haltezeit während des Temperaturprozesses [2]. Um das Konzept homogener Gegenlager aus Glas zu prüfen, wird ein erster Ansatz mit einseitigem Überlastschutz realisiert (Abb. 1 a Nr. 6). Vor der Temperaturbehandlung wird der dreilagige Stapel, der aus zwei Siliziumwafern (a, c) und einem Glaswafer (b) besteht, zueinander positioniert. Der erste laserstrukturierte Wafer (a) wird dazu als Werkzeug mit definierten Ausnehmungen genutzt. Der umzuformende Glaswafer (b) wird auf dem ersten Siliziumwafer positioniert. Die Platzierung des Drucksensorchips wird 
durch definierte Ausnehmungen im zweiten Siliziumwafer (c) vorgegeben. Wird der Waferstapel horizontal in eine Heizkammer eingebracht und auf $750{ }^{\circ} \mathrm{C}$ erwärmt, erweicht der Glaswafer (Abb. 1 b). Aufgrund des Eigengewichts des Glases sinkt es an den laserstrukturierten Ausnehmungen gezielt ein (Abb. 1 a, Nr. 2). Durch das Fügen der Wafer bei $750{ }^{\circ} \mathrm{C}$ wird eine signifikante Waferkrümmung erzeugt. Diese Waferkrümmung kann durch die Nutzung eines zweiten Siliziumwafers auf dem Glaswafer reduziert werden, was zu symmetrischen mechanischen Belastungen im Waferstapel führt und die Waferkrümmung ausgleicht (Abb. 1 b).

1

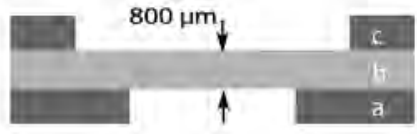

2

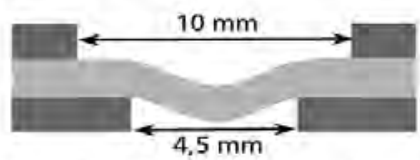

3

4
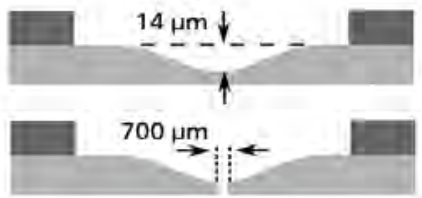

5

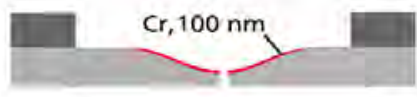

6

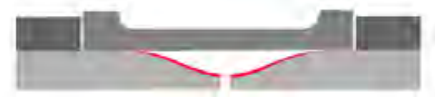

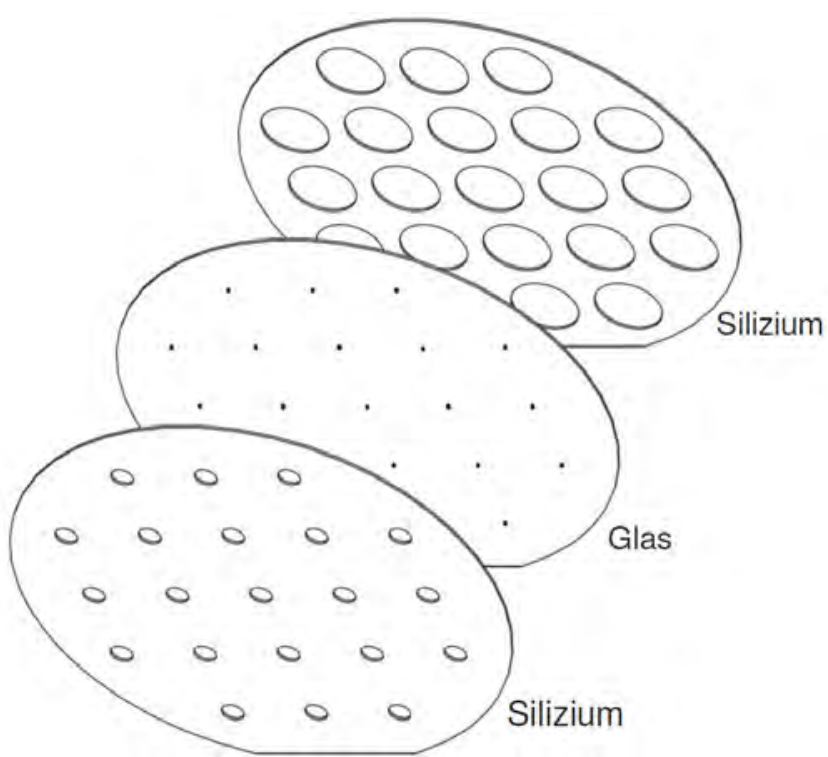

Abb. 1 Herstellung strukturierter Gegenlager.

a) Prozessschritte zur Herstellung von strukturierten Gegenlagern, b) Waferstapel vor dem Positionieren unter Reinraumbedingungen.

Die weiteren Prozessschritte erfordern das Entfernen des ersten Siliziumwafers (a) durch Läppen oder Grinden (Abb. 1 a, Nr. 3). Für den notwendigen Druckausgleich zwischen dem Gegenlager und dem Umgebungsdruck wird eine Kapillare mit einem Durchmesser von $700 \mu \mathrm{m}$ durch Ultraschallbohren eingebracht. Um das selektive Anodische Bonden der Siliziummessplatte zu realisieren, wird durch VakuumBedampfen eine $100 \mathrm{~nm}$ dünne Chromschicht strukturiert. Bereits vereinzelte Verformungskörper werden anschließend Anodisch auf das Gegenlager gebondet. Die gleichzeitige Herstellung von 21 Proben ist möglich.

Durch Wafersägen werden die Gegenlager mit Siliziummessplatte (Drucksensorchip) in quadratische Proben mit einer Kantenlägen von $15 \mathrm{~mm}$ vereinzelt. Eine wichtige Voraussetzung ist eine ebene Oberfläche, deren Oberflächenrauigkeit für das Anodische Bonden geeignet sein muss. Daher wird im Folgenden die Oberflächenqualität des Glaswafers untersucht. Die vereinzelten Proben werden in ein Testgehäuse eingebracht und der Druck $p$ wird in Stufen vergrößert. Im Berstfall kann ein Volumenstrom detektiert werden, wenn die Messplatte beschädigt wird (Abb. 2 a). Die hergestellten Proben sind mit einem mittleren statischen Überlastdruck bis 220 bar (22 MPa) belastbar (Abb. 2 b).
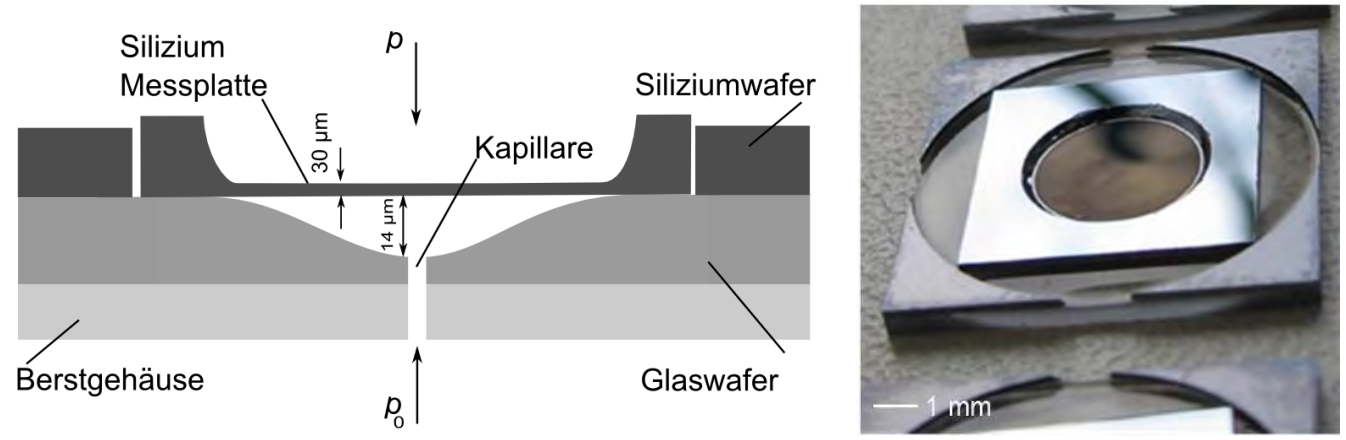

Abb. 2 Aufbau des überlastfesten Messelementes. a) Überlasttest des einseitig überlastgeschützten Silizium-Chips mit einem Gegenlager aus Glas, b) Messelement vor einem Überlasttest mit Gegenlager aus Glas, welches alle Prozessschritte beinhaltet (Anodisches Bonden, Bedampfen, Hoch-Temperatur-Umformung). 
Für eine reproduzierbare Überlastfestigkeit sind die Gegenlagertiefe, der Kapillardurchmesser der Druckausgleichsbohrung sowie eine defektfreie Glasoberfläche entscheidend (Tabelle 1). Für den Kapillardurchmesser wurde zusätzlich zum Bohrungsdurchmesser der Randbereich hinzuaddiert, in dem Fehlstellen der Oberfläche auftreten.

Tabelle 1. Reproduzierbarkeit des Kapillardurchmessers durch Ultraschallbohren sowie der Gegenlagertiefe durch thermisches Umformen eines Glaswafers.

\begin{tabular}{lll}
\hline \hline & Kapillardurchmesser & Gegenlagertiefe \\
\hline Mittelwert $\mu$ & $608 \mu \mathrm{m}$ & $12,29 \mu \mathrm{m}$ \\
Standard Abweichung $\sigma$ & $33,97 \mu \mathrm{m}$ & $1,77 \mu \mathrm{m}$ \\
\hline \hline
\end{tabular}

Die Gegenlagertiefen auf einem Wafer sind charakteristisch verteilt (Abb. 3 a). Mögliche Ursachen für die Verteilung der Gegenlagertiefen sind eine inhomogene Temperaturverteilung der Heizkammer, sowie eine Schräglage des Wafers in der Heizkammer. Das Oberflächenprofil eines einzelnen asphärischen Gegenlagers zeigt sich als kreisrunde Vertiefung in der Waferoberfläche (Abb. 3 b).
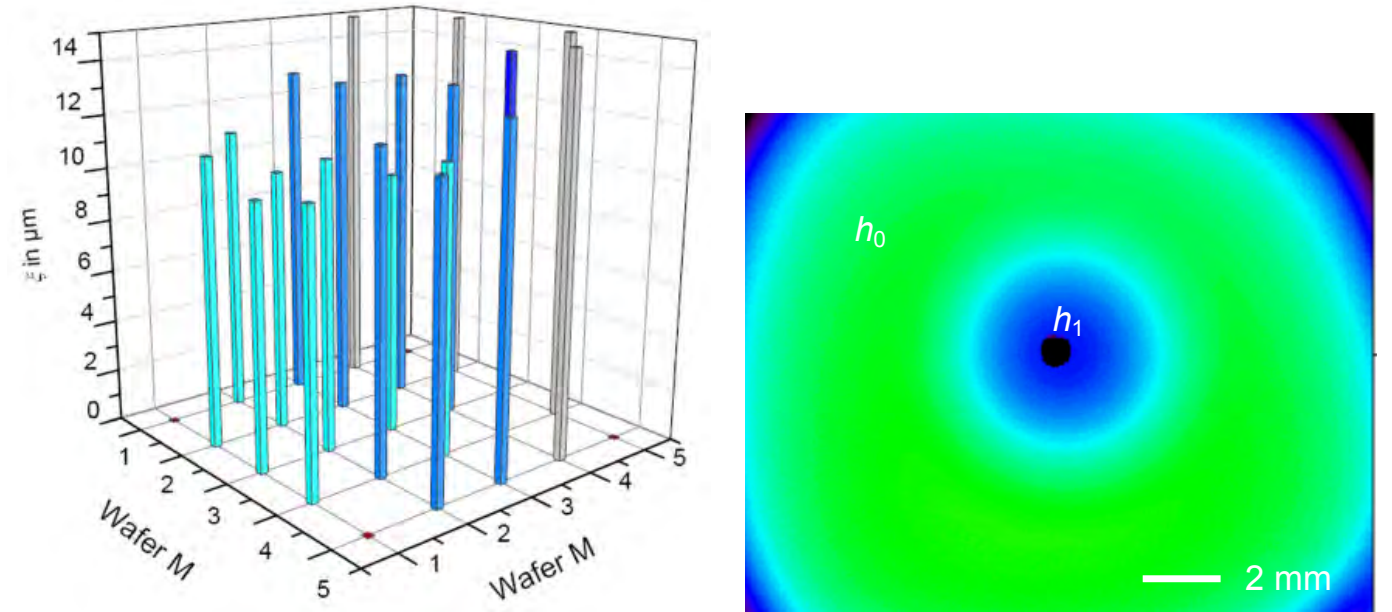

Abb. 3 Messergebnisse zum Gegenlager [3]

a) Verteilung der Gegenlagertiefe eines Wafers, b) Chromatische Interferenzanalyse der Oberfläche eines Gegenlagers $\left(h_{0}=0 \mu m, h_{1}=12 \mu m\right)$ mit Druckausgleichsbohrung.

Dabei liegt die ultraschallgebohrte Druckausgleichs-Kapillare aufgrund eines manuellen Ausrichtschrittes exzentrisch zum tiefsten Punkt $h_{1}$ des Gegenlagers. Der homogene Randbereich auf Waferebene $h_{0}$ ist für das Anodische Bonden einer Silizium-Druckmessplatte geeignet. Dazu ist es erforderlich, dass der ebene Bereich des Glasgegenlagers frei von Oberflächendefekten ist. Nach dem Temperaturprozess können durch Oberflächenanalyse mit Lichtmikroskopie vereinzelt punkförmige Unebenheiten festgestellt werden. Die Unebenheiten führen zu lokalen mechanischen Spannungsüberhöhungen während des Anodischen Bondens. Dies könnte zu Leckagen an der Fügestelle zwischen der Messplatte und dem Glasgegenlager führen. Die Unebenheiten und deren Entstehungsprinzip können durch Röntgendiffraktogramme von Glasproben, die in unterschiedlichen Prozessumgebungen getempert wurden, charakterisiert werden.

\subsection{Kristobalit-Bildung auf Glaswafern während der Ausformung}

Borofloatglas ist ein wichtiger Glastyp inorganischen Glases, welcher aus dem heutigen Technologiebereich, sei es als Substratmaterial oder als Matrix-Material für die Anwendung in Siliziumkarbid verstärkten Glasfasermaterialien, nicht mehr wegzudenken ist [4]. SCHOTT BOROFLOAT ${ }^{\circledR} 33$ weist die in Tabelle 2 gezeigte Zusammensetzung auf.

Tabelle 2: Zusammensetzung der Glaswafer für den thermischen Umformprozess [5].

\begin{tabular}{lr}
\hline \hline Oxide & Gewichtsanteil (\%) \\
\hline $\mathrm{SiO}_{2}$ & 81 \\
$\mathrm{~B}_{2} \mathrm{O}_{3}$ & 13 \\
$\mathrm{Na}_{2} \mathrm{O} / \mathrm{K}_{2} \mathrm{O}$ & 4 \\
$\mathrm{Al}_{2} \mathrm{O}_{3}$ & 2 \\
\hline \hline
\end{tabular}


Laut Hersteller besitzt das verwendet Glas eine Transformationstemperatur $T_{\mathrm{g}}=525{ }^{\circ} \mathrm{C}$, ab der die Viskosität des Glases stark zunimmt [5]. Nach Überschreiten der Erweichungstemperatur des Glases kann es beim erneuten Abkühlen zur Kristallisation einiger Bestandteile des Glases kommen. Jede Glasschmelze strebt den energieärmsten Zustand an, d.h. die Kristallisation. Wird eine Glasschmelze unendlich langsam abgekühlt, so entsteht ein vollständig durchkristallisierter Werkstoff. Die amorphen Eigenschaften des Glases werden nur erhalten, indem die Glasschmelze im Herstellungsprozess schnell unterkühlt wird. Hierdurch wird die Beweglichkeit der netzwerkwandelnden Ionen in der Schmelze stark eingeschränkt. Im Gegensatz dazu kann es bei einer nachträglichen Erwärmung des Glases über $T_{\mathrm{g}}$ ebenfalls zur Keimbildung und anschließendem Kristallwachstum kommen. In der Regel treten Kristallphasen in Gläsern als unvollständig ausgebildete Kristalle wie Dendrite, Kristallite und Sphärolite auf [6]. Beim Wachstum eines überkritischen Keims, kommt es bevorzugt zu Anlagerungen von Ionen an den Stellen im Glas, wo die höchste Anlagerungsenergie $Q$ herrscht [7]. Die bevorzugte Kristallart in SCHOTT BOROFLOAT ${ }^{\circledR} 33$ sind die Kieselsäurekristallphasen Kristobalit und Tridymit. Der Tridymit ist als Tieftemperaturmodifikation des Kristobalits zu verstehen, welcher unterhalb einer Temperatur von $870^{\circ} \mathrm{C}$ als stabile Phase vorliegt. Unterhalb einer Temperatur von $230^{\circ} \mathrm{C}$ wandelt sich der kubische $\alpha$-Kristobalit in den tetragonale $\beta$-Kristobalit um [6].

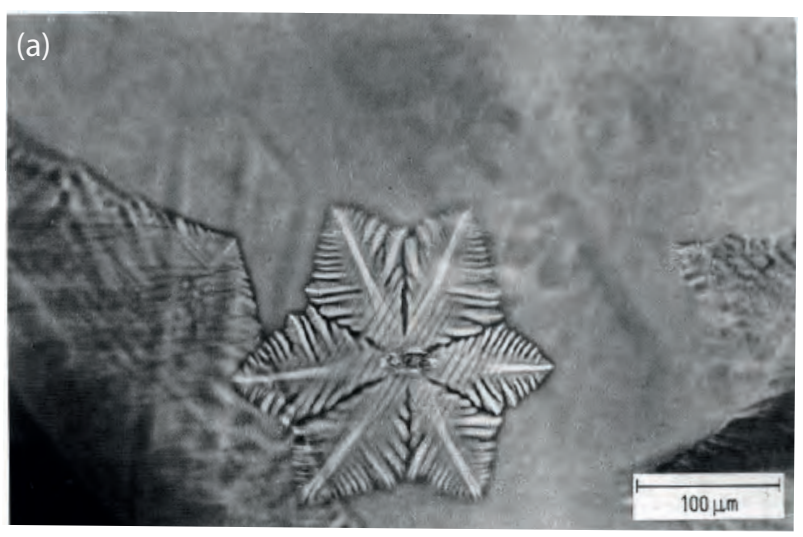

Abb. 4. Formen der Kristobalit-Kristall Ausbildung.

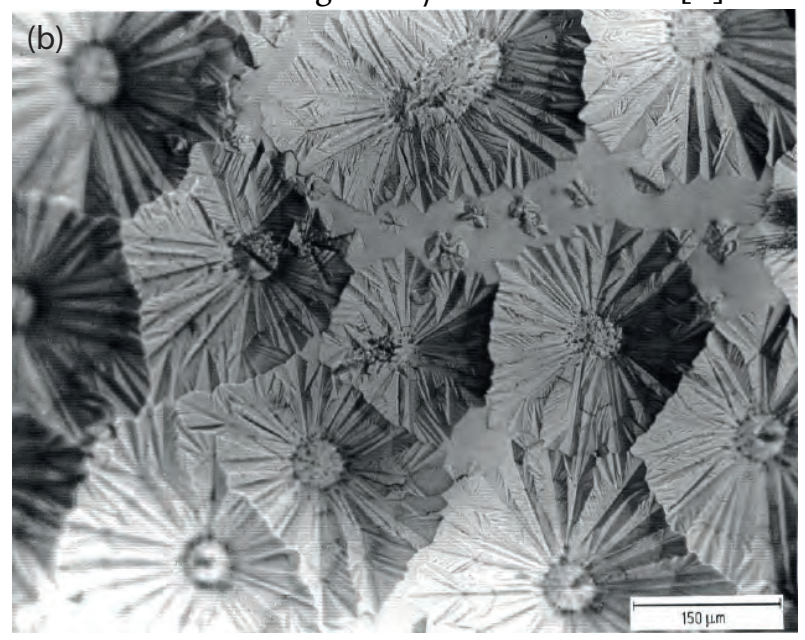

a) Ideal ausgebildeter Kristobaliteinzelkristallit, mit den typischen sechs farnähnlichen Armen, b) Kristobalitoberflächenkristallisation: Bildung von mehreren radial angeordneten Armen, um ein Keimzentrum [6].

Die in Abb. 4 gezeigten Kristallite führen durch unterschiedliche Volumenausdehnung zur Spannungsüberhöhung um jeden einzelnen Kristallit, und in letzter Konsequenz zur Rissinitiierung und dadurch zum Versagen des Bauteils. In unserem Fall zum Ausfall des überlastfesten Silizium-Differenzdrucksensors.

MoĞULKOÇ et al. [7] haben den Zusammenhang von Keimbildung und Kristobalitwachstum in Abhängigkeit von der Temperatur und Zeit in $500 \mu \mathrm{m}$ dicken Borofloatglaswafern gezeigt [7]. Hierbei wurde die Temperatur an Luft in einem Bereich von $660{ }^{\circ} \mathrm{C}$ und $850{ }^{\circ} \mathrm{C}$ variiert und die oberflächennahe Keimbildung und das Kristallwachstum mittels Lichtmikroskopie und Rasterkraftmikroskopie untersucht. Die folgende Tabelle 3 zeigt die Resultate der Studie.

Tabelle 3. Inkubationszeiten für die Keimbildung und Wachstumsraten von Kristobalit in SCHOTT BOROFLOAT ${ }^{\circledR} 33$ [7].

\begin{tabular}{|c|c|c|}
\hline Temperatur in ${ }^{\circ} \mathrm{C}$ & $\begin{array}{c}\text { Gemessene Inkubationszeit } \\
\text { der Kristallisation }\end{array}$ & $\begin{array}{c}\text { Gemessene lineare Kristall- } \\
\text { wachstumsrate in } \mu \mathrm{m} / \mathrm{h}\end{array}$ \\
\hline 660 & $23 \mathrm{~h}$ & 0,20 \\
\hline 680 & $5 \mathrm{~h}$ & 0,24 \\
\hline 700 & $90 \mathrm{~min}$ & 0,41 \\
\hline 720 & $30 \mathrm{~min}$ & 0,67 \\
\hline 740 & $5 \mathrm{~min}$ & 0,83 \\
\hline 770 & - & 1,56 \\
\hline 800 & - & 2,90 \\
\hline 820 & - & 3,55 \\
\hline 850 & - & 5,18 \\
\hline
\end{tabular}


Eine Erklärung für das Auftreten der Kristalle an der Oberfläche sieht MoĞULKOç et al.[7] in dem Kontakt zur Umgebungsluft und dem damit verbundenen Reaktionen zwischen der Luftfeuchtigkeit und Sauerstoff der Umgebung, welches die Kristallbildung begünstigt. In einem Beschichtungsexperiment mit Silizium und Siliziumnitrid konnten MoĞULKOÇ et al. [7] zeigen, dass die Kristallitbildung stark unterdrückt werden kann. Aus diesem Grund ist davon auszugehen, dass der Kontakt zur Umgebung maßgeblichen Einfluss auf das Wachstum von Kristobalit hat. Des Weiteren ist durch Arbeiten von EKSTRÖM et al.[8] und SCHÄFER et al. [9] bekannt, dass die Kristallbildung in Glas eine intrinsische Eigenschaft ist, welche durch eine Schutzschicht nicht unterdrückt sondern nur verzögert werden kann. In dieser Arbeit soll untersucht werden, ob die Unterdrückung der Keimbildung ohne Verwendung einer Schutzschicht, nur durch Änderung der Prozessumgebung, erreicht werden kann. Eine Beschichtung stellt einen weiteren Reaktionspartner für das Glas dar und es sind hierfür mindestens zwei zusätzliche Prozessschritte notwendig, die durch Beschichten und Entfernen der Passivierung entstehen.

\section{Versuchsergebnisse und Diskussion}

Alle Temperaturexperimente im Rahmen dieser Arbeit wurden an Glasproben SCHOTT BOROFLOAT ${ }^{\circledR} 33$ mit den Maßen 10 x 10 x 0,8 mm³ durchgeführt. Hierbei werden die Proben sorgfältig in Aceton und Isopropanol von Schmutz befreit, mit Druckluft getrocknet und anschließend in einem Rohrofen (horizontal, Gero, Typ F70-500, $T_{\max }=1300{ }^{\circ} \mathrm{C}$ ) auf einem Graphitblock mittig positioniert. Die Verwendung des Graphitblocks dient hierbei der leichteren Ablösbarkeit der Glasproben nach dem Temperaturschritt. Die Proben werden in Stickstoff $\left(q=1,8 \mathrm{l} / \mathrm{min}\right.$, Reinheit: 5.0) und in Vakuum $\left(p=10^{-5} \mathrm{mbar}\right)$ auf $700{ }^{\circ} \mathrm{C}$ aufgeheizt und für jeweils $5 \mathrm{~h}$ bei dieser Temperatur belassen. Zusätzlich wurde eine weitere Probe in Vakuum für $12 \mathrm{~h}$ auf $700{ }^{\circ} \mathrm{C}$ gehalten. Die Proben an Luft wurden auf eine Temperatur von $750{ }^{\circ} \mathrm{C}$ aufgeheizt. Diese Parameter orientieren sich an den Prozessparametern für die Fertigung des Differenzdrucksensors. Es wurde nach mehreren Vorversuchen die Prozesstemperatur von $750{ }^{\circ} \mathrm{C}$ auf $700{ }^{\circ} \mathrm{C}$ herabgesetzt, um die Vergleichbarkeit zu einem zuvor verwendeten Muffelofens sicherzustellen. Nach der Haltezeit kühlen die Proben im abgeschalteten Ofen in der jeweils herrschenden Atmosphäre ab. Zur Charakterisierung werden die Proben bei Raumtemperatur mittels Röntgendiffraktometrie (XRD, SmartLab, Rigaku) in einem Winkelbereich von $2 \theta=10^{\circ}-100^{\circ}$ untersucht. Die Oberflächenanalysen wurden mittels Lichtmikroskopie (LM, Olympus BX50) und Rasterkraftmikroskopie (AFM, MFP-3D Stand Alone, Asylum Research) durchgeführt.

\subsection{Temperung an Luft}

Die Untersuchung der in Luft wärmebehandelten Proben zeigt sowohl im LM als auch im AFM kreisförmige Gebilde (Abb. 5 a, b). Die AFM-Aufnahmen zeigen kreisrunde ausgedehnte Strukturen, die zur Mitte hin leicht erhöht sind. Der Durchmesser der in Abb. 5 gezeigten Struktur beträgt $10 \mu \mathrm{m}$. Es zeigt sich ein radial dentritisches Wachstum, welches bei Kristobalitkristalliten zu erwarten ist. Der Unterschied zwischen der thermischen Ausdehnung des Kristalls und der umgebenden Matrix kann groß sein, was zu induzierter thermischer Spannung führt, wenn die Probe abgekühlt wird.
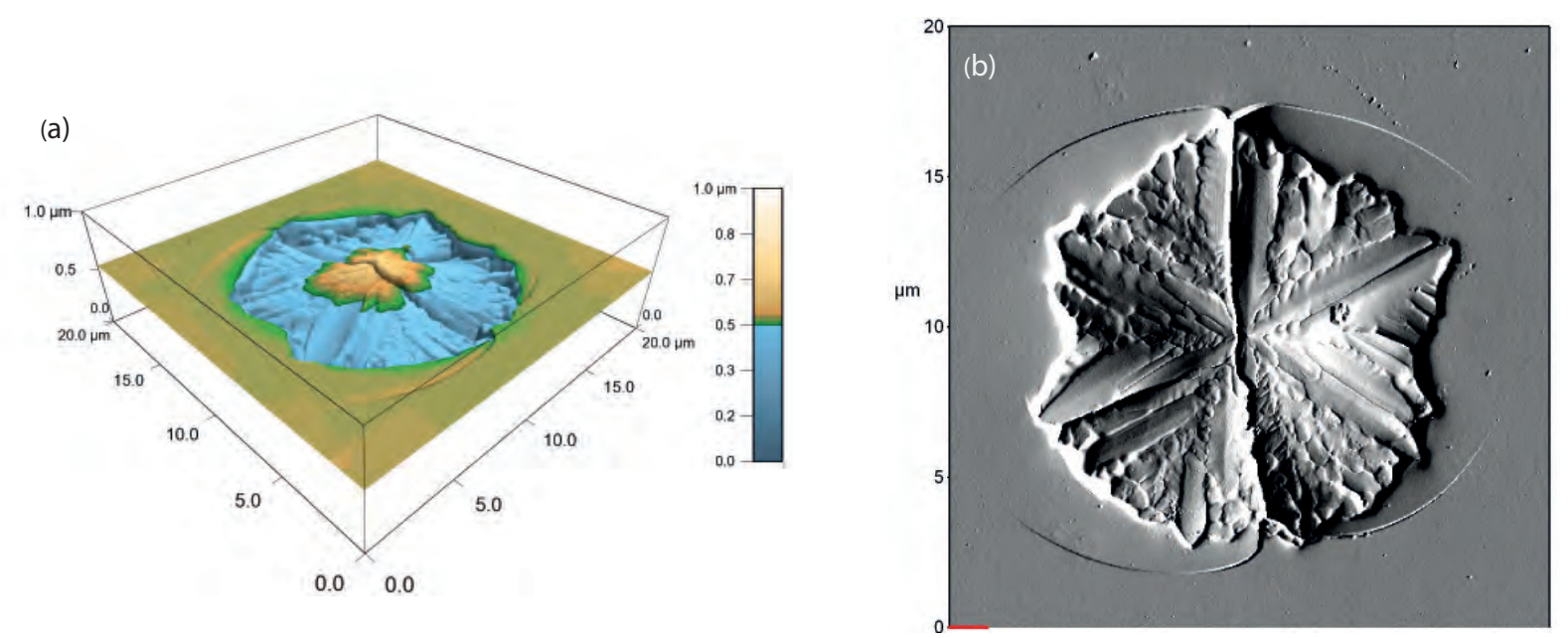

Abb. 5. Charakterisierung der Kristobalit-Struktur.

a) 3D-AFM-Aufnahme in einem Scanbereich von $20 \times 20 \mu \mathrm{m}^{2}$ eines Sphärolits, b) Aufsichtperspektive von (a). 
Beim zusätzlichen $\alpha / \beta$ - Kristobalitübergang erfolgt eine Volumenschrumpfung von 3,9 \% durch die Änderung der Struktur von kubisch zu tetragonal [7]. Diese Spannungsüberhöhung tritt lokal um die Kristobalitkristalle auf, erkennbar an den kreisrunden Linien in Abb. 5, und kann in der Größenordnung von einigen GPa liegen [4]. Dies ist ausreichend für die spontane Rissbildung an dieser Stelle und dem damit verbundenen Versagen, dem Bruch des Glases.

Im Vergleich mit den ermittelten Wachstumsraten von MoĞULKoç et al. (Tabelle 2), liegt die lineare Wachstumsrate bei einer Temperatur von $750{ }^{\circ} \mathrm{C}$ bei ca. $1,07 \mu \mathrm{m} / \mathrm{h}$, dies hätte einen Kristallitradius von ungefähr 5,36 $\mu \mathrm{m}$ zur Folge, was in guter Übereinstimmung mit den erhaltenen Ergebnissen in Abb. 5. steht. Zur weiteren Charakterisierung der kristallinen Strukturen wurde ein $2 \theta / \omega$-Scan mittels Röntgendiffraktometrie durchgeführt, dessen Ergebnis in Abb. 6 gezeigt ist.

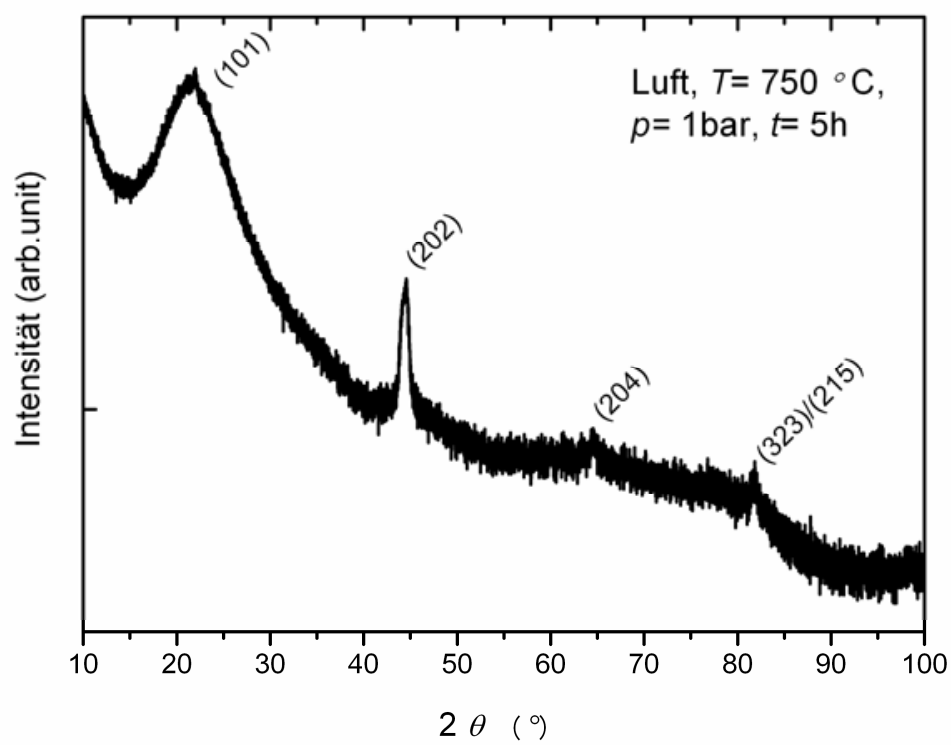

Abb. 6. 2 $\theta / \omega$-Scan der an Luft getemperten Probe. Die auftretenden Reflexe sind dem B- Kristobalit zuzuordnen.

Im Röntgendiffraktogramm der an Luft getemperten Probe tritt ein breiter Reflex zwischen $15^{\circ}$ und $25^{\circ}$ auf, welcher dem amorphen Glas zugeordnet werden kann. Des Weiteren treten 4 Reflexe auf, die dem $\beta$ Kristobalit zuzuordnen sind (ICDD-PDF 04-012-1126).

\subsection{Temperung an Stickstoff}

Wirkt die Umgebungsluft und der damit verbundene Sauerstoff begünstigend auf das Wachstum von Kristobalit, wie von Moğulkoç et al. vorgeschlagen, sollte dieses durch die Verwendung eines Inertgases, wie $\mathrm{N}_{2}$ unterdrückt werden. In Abb. 7 ist die AFM-Aufnahme der Glasprobe gezeigt, welche 5 h, bei $700{ }^{\circ} \mathrm{C}$ in Stickstoff getempert wurde.
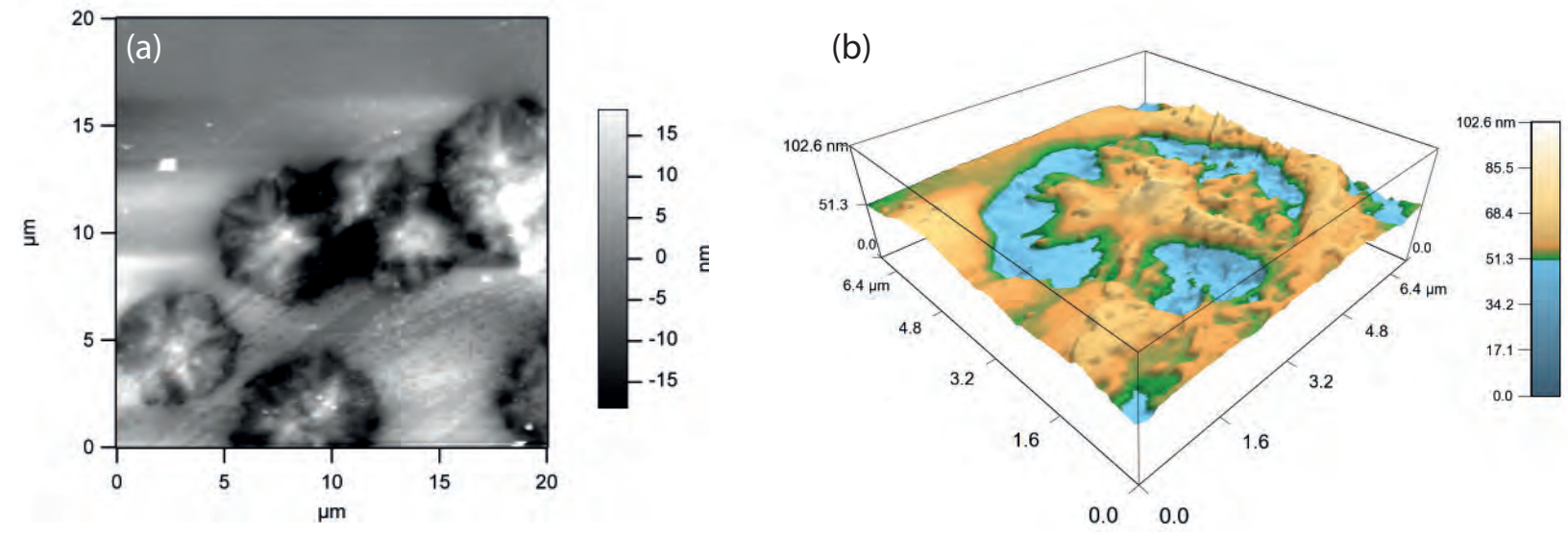

Abb. 7.Oberflächenstruktur der bei $700{ }^{\circ} \mathrm{C}$ in Stickstoff getemperten Proben.

a) AFM-Aufnahme mehrerer Kristobaltkristalle auf der in $\mathrm{N}_{2}$ ausgelagerten Probe, b) 3D-Aufnahme eines in (a) vorhandenen Kristallits. 
Im Unterschied zu den Proben, die an Luft getempert wurden, treten die Kristobalitkristalle jetzt in Gruppen auf und sind weniger stark ausgeprägt. Der Durchmesser der einzelnen Kristallite beträgt im Durchschnitt $5 \mu \mathrm{m}$, also die Hälfte der Größe der Kristallite, die an Luft entstehen. Im Vergleich mit Tabelle 3 wird deutlich, dass das Wachstum einer Temperatur von ca. $700{ }^{\circ} \mathrm{C}$ entspricht, obwohl die in Tabelle 3 gezeigten Werte für eine sauerstoffhaltige Atmosphäre gelten. Trotz der Verwendung eines Inertgases kommt es zum Wachstum von Kristobalit, was darauf schließen lässt, dass die Umgebungsluft nicht allein den Grund für die Ausbildung der Kristallite darstellt, was die Theorie, der intrinsischen Eigenschaft der Kristallitbildung des Glases bestätigt. Das in Abb. 8 gezeigte Röntgendiffraktogramm der Probe bestätigt das Wachstum von Kristobalit.

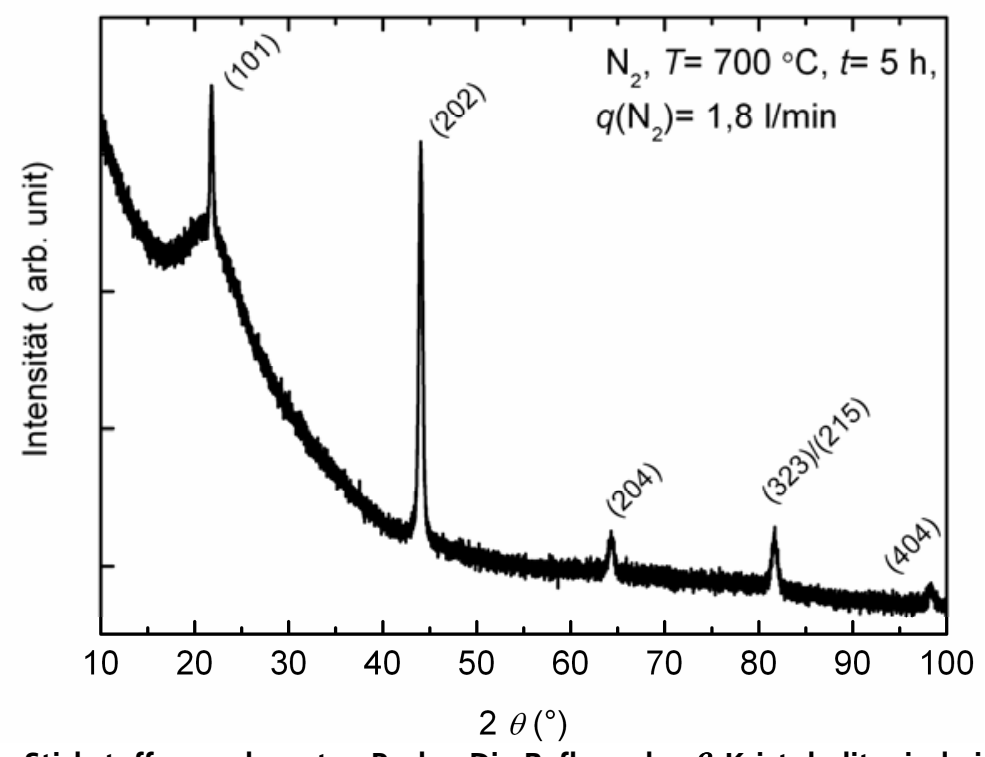

Abb. 8. 2 $\theta / \omega$-Scan der in Stickstoff ausgelagerten Probe. Die Reflexe des $\beta$ - Kristobalits sind eindeutig indizierbar.

Im Vergleich zum Röntgendiffraktogramm der an Luft ausgelagerten Probe fällt auf, dass eine stärkere Ausprägung der einzelnen Reflexe vorliegt. Im Winkelbereich von $15^{\circ}$ bis $25^{\circ}$ ist der amorphe Beitrag des Glases zum Untergrund zu identifizieren. Am Beispiel des (101)-Reflexes lässt sich gut der oben beschriebene Unterschied zur Probe an Luft erkennen. Das Verhältnis des (101)-Reflex zum (202)-Reflex unterscheidet sich zu der Probe, welche an Luft ausgelagert wurde. Bei der in $\mathrm{N}_{2}$ ausgelagerten Probe beträgt es 0.92 wohingegen das Verhältnis der an Luft ausgelagerten Probe 0.42 beträgt. Daraus lässt sich ableiten, dass das Kristallitwachstum in (202)-Richtung weiter begünstigt wird.

Das Vorhandensein von Kristobalit trotz Verwendung eines Schutzgases lässt den Schluss zu, dass anders als in [7] postuliert der Sauerstoff und die Luftfeuchte in der Umgebungsluft nicht maßgeblich für das Wachstum von Kristobalit verantwortlich sind. Viel mehr ist in der Affinität zur Diffusion von Silizium und Sauerstoff der Grund für das Wachstum der Kristalle zu sehen. Es ist aus früheren Experimenten von ScHÄFER et al.[9] bekannt, dass $\mathrm{SiO}_{2}$ in Vakuum ein sehr flüchtiger Bestandteil ist, der als $\mathrm{SiO}$ und $\mathrm{O}_{2}$ in der Gasphase vorliegt. Durch eine Arbeit von EKSTRÖM et al. [8], in der das Wachstum von Kristobalit und die Umwandlung $\mathrm{zu}$ amorphem $\mathrm{SiO}_{2}$ untersucht wurden, geht hervor, dass die Kristobalitkristalle über die Gasphase entstehen. Es ist darauf hinzuweisen, dass das Gasphasenwachstum ein sehr komplexer Vorgang ist, wobei die Anwesenheit von Wasser, Sauerstoff oder Wasserstoff sicherlich deutlichen Einfluss auf die Kinetik des Wachstumsprozesses hat. Durch die Diffusion und die Evaporation von $\mathrm{SiO}_{2}$ aus der Probe zur Oberfläche, lässt sich das Wachstum von Kristobalit durch die Anwesenheit eines Stickstoffflusses nicht unterdrücken. Es kann davon ausgegangen werden, dass der örtlich überhöhte Anteil von gasförmigen $\mathrm{SiO}_{2}$ ausreichend ist, um das Wachstum von Kristobalit in der Zeit der Wärmebehandlung zu begünstigen.

\subsection{Temperung unter Vakuum}

Die Proben, welche für $12 \mathrm{~h}$ im Vakuum ausgelagert wurden, zeigen ein sehr raues Bild der Oberfläche. Es ist davon auszugehen, dass durch die Eigenschaft des $\mathrm{SiO}_{2}$ in der Gasphase ebenfalls ein Wachstum von Kristalliten unter Vakuum zu erwarten ist. Im Gegensatz zum Experiment mit Stickstoff ist es, wegen des nicht vorhandenen Gasdrucks im Ofen wahrscheinlich, dass das Wachstum durch die vermehrte Evaporation von $\mathrm{SiO}_{2}$ begünstigt wird. Entgegen dieser Erwartung zeigt das in Abb. 10 (a) dargestellte Röntgendiffraktogramm der für $5 \mathrm{~h}$ getemperten Probe keinerlei Reflexe des Kristobalits. Im Winkelbereich zwischen $15^{\circ}$ und $25^{\circ}$ ist ausschließlich der amorphe Beitrag des Glases zu erkennen. 
Oberflächenanalysen mittels LM und AFM (Abb. 9) bestätigen die Abwesenheit von Kristobalitkristalliten auf der Oberfläche der Glasprobe. Die gemessene mittlere Oberflächenrauhigkeit liegt bei RMS $=3,6 \mathrm{~nm}$. Die Reflexe im Diffraktogramm (Abb. 10(b)) der Probe, welche für $12 \mathrm{~h}$ behandelt wurde, sind eindeutig der kristallinen Phase des Kristobalits zuzuordnen. Im Vergleich zu den Proben in Stickstoff und an Luft, sind die Reflexe noch stärker ausgeprägt und weitere Orientierungen treten auf. Das Intensitätsverhältnis von $I(202) / I(101)$ liegt bei 0.42, ähnlich der Probe an Luft.

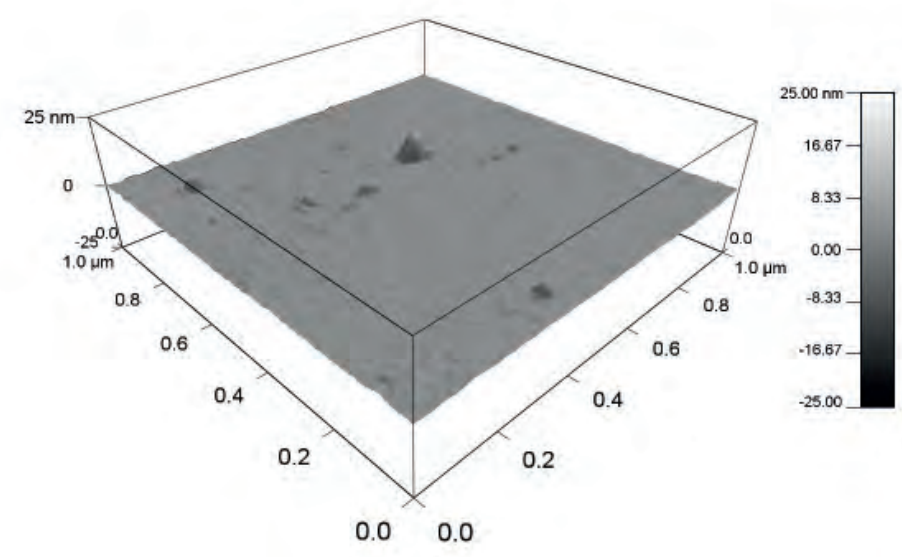

Abb. 9. AFM- Aufnahme der Oberfläche der Größe $1 \times 1 \mu \mathrm{m}^{2}$, der für $5 \mathrm{~h}$ in Vakuum getemperten Glasprobe.
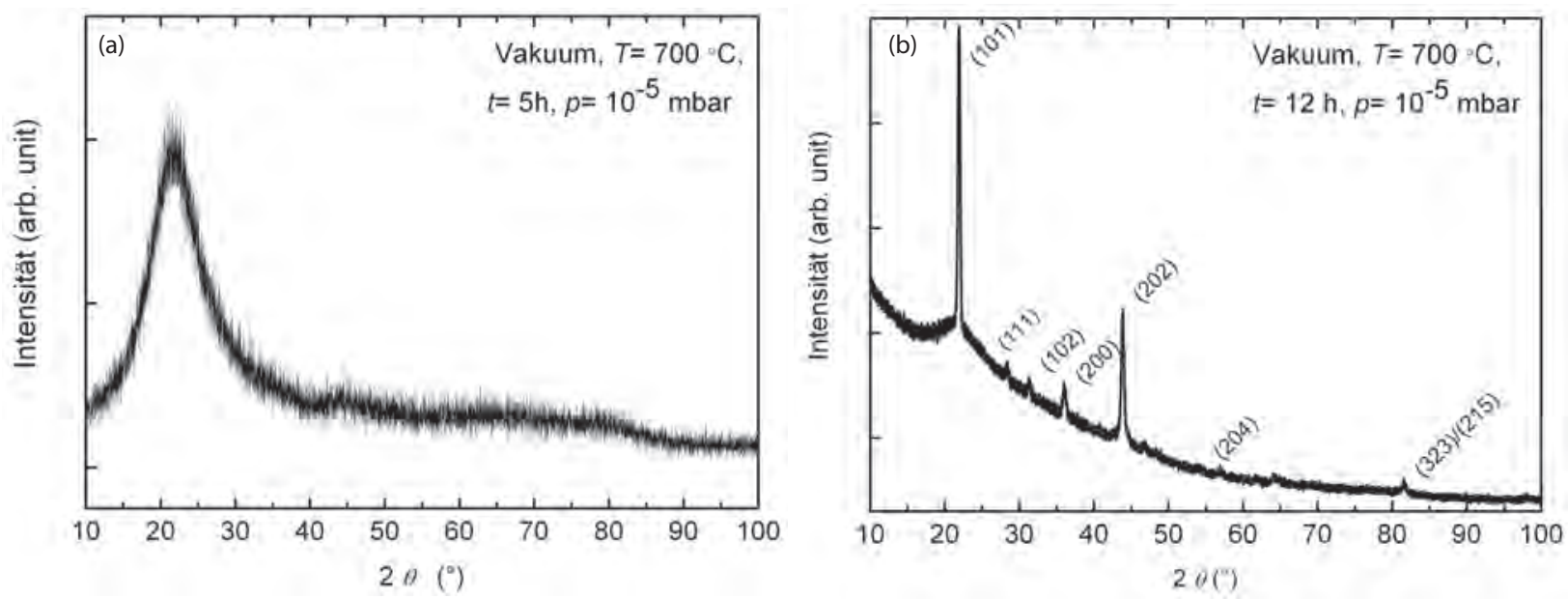

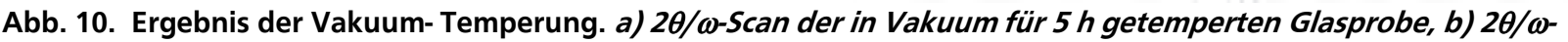
Scan der in Vakuum für 12 h getemperten Glasprobe.

Abb. 11 zeigt die Glasoberfläche der Probe, welche für $12 \mathrm{~h}$ im Vakuum behandelt wurde. Es sind eine Vielzahl von Kristalliten zu erkennen, die teilweise bevorzugt in horizontaler Richtung dicht nebeneinander gewachsen sind. Diese Linien sind sehr wahrscheinlich auf das Polieren von Herstellerseite zurückzuführen. Sie stellen für die Keimbildung von Kristobalit ideale Defekte dar. Der Durchmesser der einzelnen Kristallite variiert stark von 3,3 $\mu \mathrm{m}$ (Abb. 11(b)i) in den aneinander gereihten Kristalliten bis hin zu $7 \mu \mathrm{m}$ (Abb. 11(b)ii), wenn ein Kristall frei auf der Oberfläche gewachsen ist. Dies entspricht einer Wachstumsrate von $0,29 \mu \mathrm{m} / \mathrm{h}$, die wiederum einer Temperatur von ca. $690{ }^{\circ} \mathrm{C}$ (vgl. Tabelle 3) an Luft entsprechen würde. Die Inkubationszeit für die Keimbildung liegt in diesem Temperaturbereich zwischen 90 min und 5 h. Daher wäre zu erwarten, dass Kristobalit ebenfalls nach 5 h in Vakuum auftreten sollte. Es ist allerdings zu berücksichtigen, dass die Transportprozesse des $\mathrm{SiO}_{2}$ bei diesen Temperaturen verlangsamt stattfinden, so dass es einer längeren Zeit bedarf, um lokal $\mathrm{SiO}_{2}$ in die Gasphase übertreten zu lassen, was schließlich auch im Vakuum zum Wachstum von Kristobalit führt. EKSTRÖM et al. [8] hatte bereits auf den maßgeblichen Einfluss der Temperatur auf das Kristobalitkeimbildung und -wachstum hingewiesen, obgleich die damaligen Experimente in einem höherem Temperaturbereich zwischen $820{ }^{\circ} \mathrm{C}$ und $1100{ }^{\circ} \mathrm{C}$ durchgeführt worden sind. Der stetige Abtransport des Gases durch das Evakuieren des Ofenrohrs und die niedrigere Arbeitstemperatur könnten mögliche Ursachen für das verzögerte Kristallwachstum im Vakuum darstellen. 

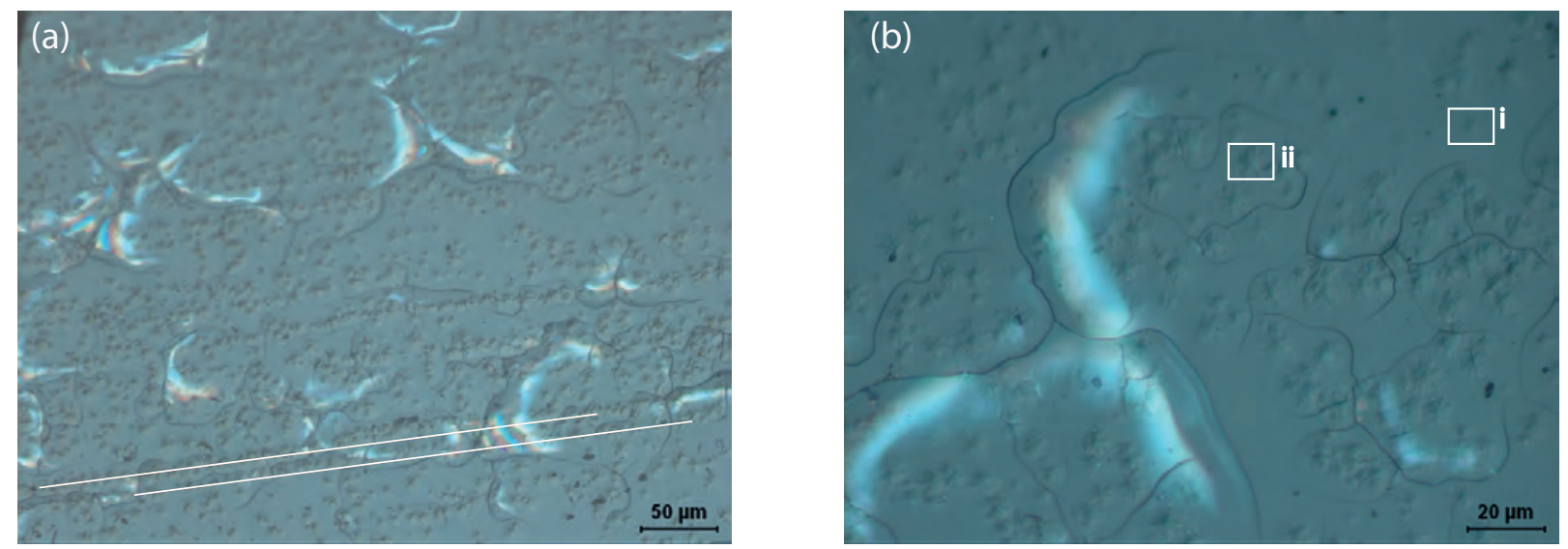

Abb. 11. LM- Aufnahmen der Glasoberfläche der im Vakuum für $12 \mathrm{~h}$ behandelten Proben.

Eine weitere Tatsache, welche in Abb. 11 deutlich wird, ist die vermehrte Rissbildung der Probe. Die gesamte Probe ist von Mikrorissen durchzogen, welche durch das Vorhandensein einer Vielzahl von Kristobalitkristallen, hervorgerufen werden. Sie werden durch die unterschiedliche Volumenausdehnung im Vergleich zum amorphen Teil der Probe verursacht und lassen auf eine erhöhte Instabilität des Glases schließen.

\section{Fazit}

Es wurde gezeigt, dass das Kristobalitwachstum in SCHOTT BOROFLOAT ${ }^{\circledR} 33$ bei einer Temperatur von $700{ }^{\circ} \mathrm{C}$ bei einer Haltezeit von $5 \mathrm{~h}$ im Vakuum ( $\left.p=10^{-5} \mathrm{mbar}\right)$ unterdrückt werden kann. Sowohl in Luft als auch in Stickstoff bildet sich Kristobalit dagegen innerhalb von $5 \mathrm{~h}$ aus. Wird die Haltezeit im Vakuum auf 12 h erhöht, kommt es auch zu einer vermehrten Kristallisation des Kristobalits über der gesamten Oberfläche der Probe. Das heißt, dass der Prozessschritt der Herstellung von homogenen Gegenlagern aus Glas im Vakuum, eine sinnvolle Alternative zur Verwendung von Schutzschichten darstellt und sich aus diesem Grund gut in den Herstellungsprozess integrieren lässt. Die Oberflächenbeschaffenheit des Glases ist durch die Vakuumbehandlung für den weiteren Bondingschritt geeignet und kann ohne weiteren Arbeitsaufwand verwendet werden. Das Konzept kann erweitert werden, so dass ein symmetrisches Design entsteht, welches einen beidseitigen Überlastschutz für statische und dynamische Druckspitzen sicherstellt.

\section{Danksagung}

Die Autoren danken dem Fachgebiet Oberflächenforschung, Fachbereich Material-und Geowissenschaften Technische Universität Darmstadt, für die freundliche Unterstützung sowie für die Nutzung des verwendeten Rasterkraftmikroskops.

\section{Literatur}

[1] Kober T, Wertschützky R. Mikromechanischer Überlastschutz für Drucksensoren durch strukturierte Gegenlager aus Glas. Sensoren und Messsysteme 2010. 2010.

[2] Kober T, Werthschützky R. Overload-Resistance Pressure Sensors in the Nominal range of 10 mbar (1kPa). Proceedings Eurosensors XXIV Conference, Linz, Austria. 2010.

[3] Kober T, Werthschützky R. Überlastfeste Silizium-Differenzdrucksensoren mit thermisch strukturierten Gegenlagern aus Glas. Sensoren und Messsysteme, 2012. 2012.

[4] D. Shin, Auh K, Knowles K. SiC fiber/Borosilicat Glass Composite (Partl). Journal of the Ceramic Society of Japan. 1995;103:319.

[5] Schott. Technisches Datenblatt von SCHOTT BOROFLOAT® 33; 2011.

[6] Vogel W, ed. Glasfehler: Springer Verlag 1993.

[7] Moğulkoç B, Knowles KM, Jansen HV, Ter Brake HJM, Elwenspoek MC. Surface Devitrification and the Growth of Cristobalite in Borofloat ${ }^{\circledR}$ (Borosilicate 8330) Glass. Journal of the American Ceramic Society. 2010;93(9):2713-9.

[8] Ekström T, Tilley RJD. The formation of cristobalite in evacuated silica ampoules and the transformation of cristobalite to amorphous silica. Journal of Crystal Growth. 1977;38(2):197-205.

[9] Schäfer H, ed.: Chemical Transport Reactions, Academic Press, New York 1964. 